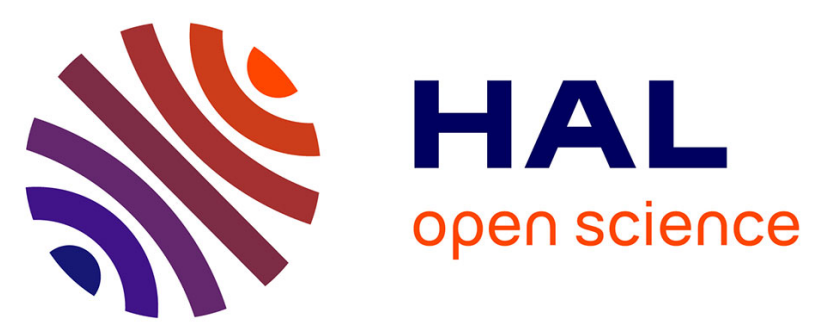

\title{
Utilisation de la spectrométrie infrarouge pour une quantification rapide du taux d'humidité dans des fibres végétales
}

Amandine Célino, Olivier Gonçalves, Frédéric Jacquemin, Sylvain Fréour

\section{- To cite this version:}

Amandine Célino, Olivier Gonçalves, Frédéric Jacquemin, Sylvain Fréour. Utilisation de la spectrométrie infrarouge pour une quantification rapide du taux d'humidité dans des fibres végétales. Revue des composites et des matériaux avancés = Journal of Composite and Advanced Materials, 2014, 24 (1), pp.81 - 95. 10.3166/rcma.24.81-95 . hal-01007416

\author{
HAL Id: hal-01007416 \\ https://hal.science/hal-01007416
}

Submitted on 22 Oct 2016

HAL is a multi-disciplinary open access archive for the deposit and dissemination of scientific research documents, whether they are published or not. The documents may come from teaching and research institutions in France or abroad, or from public or private research centers.
L'archive ouverte pluridisciplinaire HAL, est destinée au dépôt et à la diffusion de documents scientifiques de niveau recherche, publiés ou non, émanant des établissements d'enseignement et de recherche français ou étrangers, des laboratoires publics ou privés.

\section{(c)(1)}

Distributed under a Creative Commons Attribution| 4.0 International License 


\section{Utilisation de la spectrométrie infrarouge pour une quantification rapide du taux d'humidité dans des fibres végétales}

\section{Amandine Célino ${ }^{1}$, Olivier Gonçalves ${ }^{2}$, Frédéric Jacquemin ${ }^{1}$, Sylvain Fréour ${ }^{1}$}

1. Institut de Recherche en Génie Civil et Mécanique (UMR CNRS 6183)

Université de Nantes - Ecole Centrale de Nantes

IUT de Saint-Nazaire, 58 rue Michel Ange, 44600 Saint-Nazaire, France

amandine.celino@univ-nantes.fr

2. GEPEA-UMR CNRS 6144

Université de Nantes

CRTT, 37 boulevard de l'Université, BP 406, 44600 Saint-Nazaire, France

olivier.goncalves@univ-nantes.fr

RESUME. Les fibres végétales sont une alternative possible aux fibres de verre utilisées classiquement comme renforts dans certains matériaux composites. En plus d'avantages environnementaux, leur faible densité leur confère des propriétés mécaniques spécifiques élevées. Cependant, leur comportement hydrophile marqué entraîne une forte absorption d'eau lors d'une utilisation en environnement humide, ce qui peut modifier leurs propriétés mécaniques. La compréhension de ces mécanismes d'absorption d'humidité est donc indispensable à leur maîtrise. Dans ce travail, l'absorption d'eau a été étudiée par spectroscopie infrarouge à transformée de Fourier sur des fibres de lin, chanvre et sisal. Ces fibres ont été soumises à des conditions hygrométriques contrôlées. Les spectres infrarouges des échantillons ont été enregistrés en mode réflexion totale atténuée. Les informations spectrales ont permis de mettre en évidence les fonctions chimiques impliquées dans les mécanismes d'absorption de l'humidité et de quantifier le taux d'humidité retenu par les fibres. Pour cela, des modèles de régression partielle des moindres carrés ont été développés en exploitant des données expérimentales issues de mesures gravimétriques. Ces modèles se sont révélés de bons outils pour la caractérisation des cinétiques de diffusion de l'eau dans ces différentes fibres.

ABSTRACT. In the field of composite materials, natural fibres appear to be a viable replacement for glass fibres. However, in humid conditions, strong hydrophilic behavior of such materials can lead to high level of moisture absorption. This feature can result in a structural modification of the fibres and a modification of their own mechanical properties as well as those of the composites they are fitted in. Then, understanding moisture sorption mechanisms in these materials is an important issue for their efficient use. In this work, water absorption on three natural fibres (flax, hemp and sisal) was 
studied qualitatively and quantitatively using non-invasive approach, i.e. Fourier transformed infrared spectroscopy. Fibres with different moisture content were prepared using climatic chambers, allowing strictly controlled hygrothermal ageing. The equilibrium water content were assessed by gravimetric measurement and determined for each relative humidity conditions (Relative humidity ranging from $30 \%$ until $97 \%$ ). The principal chemical functions of fibres involved in the water absorption phenomenon were thereby identified and the water content of tested fibres was determined using a Partial Least Square Regression approach. The typical sorption isotherm curves described by Park model were clearly fitted, confirming the validity of our predictive model. Finally, this model has been applied for monitoring the water diffusion.

MoTs-CLES: fibres végétales, diffusion, spectroscopie infrarouge à transformée de Fourier, régression partielle des moindres carrés.

KEYWORDS: natural fibres, water sorption, Fourier transformed infrared spectroscopy, partial least square regression

\section{Extended abstract}

Nowadays, natural fibres are considered as a good alternative to glass fibres in the purpose of composite material reinforcement (particularly in automotive manufacturing or sport and leisure sector). These fibres present a better environmental impact than glass fibres (recyclability, biodegradability) as well as higher specific mechanical properties because of their low density. However, their pronounced hydrophilic behavior -due to their particular structure- leads a strong moisture absorption in wet environments. This could result in the structural modification of the fibres and an evolution of their mechanical properties together with the composites in which they are fitted in. Then, the understanding of their moisture absorption mechanisms is mandatory for a better control of such new biomaterials.

Generally, one of the most important factors controlling the water diffusion phenomenon in polymeric materials is the molecular interaction occurring between the diffusing compound and the substrate. The diffusion phenomenon is subjected to the ability of the polymer molecular sites to establish hydrogen bonds with the water molecules. Spectroscopic techniques such as Nuclear Magnetic Resonance (NMR), dielectric or Fourier Transform Infra-Red spectroscopy (FTIR) have been proved to be well adapted to study this phenomenon, since they allow the characterization of molecular interactions involving potential sorption sites for water. Among these approaches, FTIR spectroscopy has been widely used to study water transport in epoxy resins. Indeed, this technique provides attractive features: i.e. the very high sampling rate, the sensitivity, the accuracy of the quantitative analysis and the information at the molecular level contained in the vibrational spectra. Moreover, the development of Attenuated Total Reflectance FTIR spectroscopy (ATR-FTIR) encouraged and facilitated the use of this non-invasive technique directly onto solid materials. 
The aim of this work is the use of FTIR as an experimental tool to investigate the water sorption onto raw plant fibres, known to be good candidates for the reinforcement of composite materials (i.e. flax, hemp and sisal). First FTIR spectral signature of each fibre was investigated in order to describe the molecular effect of the water sorption mechanisms. Secondly, a multivariate model linking water sorption and the whole FTIR spectra was developed using partial least square regression (PLS-R). This model was applied for the accurate monitoring of the water diffusion in the tested biomaterials. Finally, the effect of water on the crystallinity of fibres has been investigated.

According to our results hydroxyl and carboxyl molecular sites of the fibre's polymer are directly impacted by the water uptake, confirming the studies achieved on less structured cellulosic material. The FTIR PLS-R models developed in order to quantify the fibre water content, showed very good agreement with the reference values (gravimetric measurements). They were used to monitor accurately sorption isotherms whereas univariate FTIR models could not fit it correctly. The proposed approach also supplied readily interpretable kinetic data by monitoring continuously the water desorption. A structural modification of the fibres after moisture sorption has also been proved by the determination of crystallinity index in hydrated samples.

\section{Introduction}

Largement exploitées dans le domaine textile, les fibres naturelles d'origine végétale représentent une alternative aux fibres de verre pour le renforcement de matrices polymériques. À l'heure du «développement durable» et de la promotion des produits biosourcés, ces fibres attirent l'attention des industries automobile, navale ou même aéronautique (Suddell et Evans, 2005). En effet, il s'agit d'une ressource naturelle abondante, à faible impact écologique et dotée de très bonnes propriétés mécaniques spécifiques (Wambua et al., 2002). Leur comportement hydrophile marqué conduit cependant à une forte absorption en milieu humide (Célino et al., 2013). Ceci modifie les dimensions et les propriétés mécaniques de ces fibres ainsi que celles des composites qu'elles renforcent (Dhakal et al., 2007 ; Placet et al., 2012 ; Symington et al., 2009). Une meilleure connaissance des mécanismes d'absorption de l'eau dans ces matériaux naturels présente donc un intérêt majeur pour une meilleure maîtrise de leur utilisation en tant que renfort dans les matériaux composites.

L'un des facteurs clés contrôlant le phénomène de diffusion de l'eau dans les matériaux et particulièrement dans les fibres naturelles est l'interaction des molécules d'eau avec le réseau macromoléculaire du substrat. Ceci est directement relié à la capacité du matériau d'établir des liaisons hydrogène avec les molécules d'eau, via sa structure moléculaire. Les techniques expérimentales permettant de mettre en évidence ces sites spécifiques et ces phénomènes d'absorption d'humidité sont la résonance magnétique nucléaire (RMN), la spectroscopie diélectrique et la spectroscopie infrarouge à transformée de Fourier (IRTF) (Mijovic et Zhang, 2003; Popineau et al., 2005). Cette dernière a été 
largement utilisée, notamment pour étudier les phénomènes de diffusion de l'eau dans les résines époxydes (Cotugno et al., 2001 ; Feng et al., 2004 ; Musto et al., 2000). Depuis quelques années, le développement de la technique de réflexion totale atténuée couplée à la spectroscopie infrarouge (ATR-IRTF) permet, en plus, l'analyse d'échantillons solides.

Peu d'études en spectroscopie IRTF ont été consacrées aux matériaux cellulosiques. Laity et Hay ont tracé la cinétique de sorption de l'eau dans des films de cellophane en enregistrant des spectres en mode réflexion (Laity et Hay, 2000). Olsson et Salmén ont étudié l'interaction de l'eau avec de la pâte à papier, mettant clairement en évidence la modification de certaines bandes d'absorption due à la présence d'eau (Olsson et Salmén, 2004). Ils établissent une relation linéaire entre l'aire de ces bandes caractéristiques et la quantité d'eau. Ces deux études proposent ainsi des modèles univariés pour quantifier la fraction d'eau contenue dans les échantillons. Cependant, ces modèles échouent à reproduire les isothermes d'absorption obtenues par d'autres techniques expérimentales.

Dans ce travail, on s'intéresse à l'absorption d'humidité dans trois types de fibres naturelles : lin, chanvre, sisal. Premièrement, les spectres IR obtenus pour des fibres contenant différentes teneurs en eau ont été analysés qualitativement. L'objectif est de montrer les bandes impactées par l'humidité afin d'identifier les sites préférentiels d'absorption des molécules d'eau dans ces matériaux. Dans un second temps, un modèle multivarié a été développé en utilisant une régression partielle des moindres carrés (PLS-R). Au contraire de l'approche univariée, cette méthode permet de relier la quantité d'eau présente dans une fibre à l'ensemble de son spectre infrarouge Après validation, le modèle a été utilisé pour tracer de façon précise la cinétique de séchage des fibres. Enfin, l'évolution de la structure cristalline des fibres en fonction de l'humidité a été étudiée.

\section{Matériaux et méthodes}

\subsection{Matériaux}

Cette étude est consacrée aux fibres de lin, de chanvre et de sisal. Parmi les fibres envisagées comme renforts pour les matériaux composites, elles possèdent les propriétés requises pour concurrencer les fibres de verre (Summerscales $e t$ al., 2010 ; Wambua et al., 2003). Les fibres de lin et de chanvre sont dites libériennes (extraites de la tige des plantes). Les fibres de sisal sont extraites de la feuille de la plante. Leur structure est multi-échelle. Dans la suite de cette étude, on travaille à l'échelle du faisceau de fibres. Un faisceau est constitué de quelques dizaines de fibres unitaires principalement composées de cellulose, hémicellulose et lignine. Ces fibres unitaires sont liées entre elles par les pectines et les cires de la lamelle mitoyenne. La cellulose semi cristalline arrangée en microfibrilles confère à ces fibres leurs propriétés mécaniques intéressantes. 


\subsection{Protocole de vieillissement humide}

Les fibres étudiées sont conditionnées par séchage au dessiccateur à température ambiante. À partir de cet état de référence, les échantillons ont ensuite été placés dans différentes ambiances humides (30, 40, 50, 60, 75, 85 et $97 \%$ d'humidité relative) afin de représenter leur cinétique de diffusion en fonction de leur teneur en eau à saturation. Un suivi gravimétrique périodique a permis de tracer la courbe de la reprise en eau des échantillons en fonction du temps (équation 1). Ceci permet de déterminer la capacité maximale d'absorption d'humidité en fonction de l'humidité relative et de tracer les isothermes de sorption de chaque fibre. On considère la saturation atteinte lorsque la masse devient stable. Cette teneur en eau à l'équilibre est une donnée d'entrée du modèle de quantification décrit dans la section suivante.

$$
M_{e}(\%)=\frac{M(t)-M_{0}}{M_{0}} \times 100
$$

où $\mathrm{M}(\mathrm{t})$ correspond à la masse de l'échantillon à l'instant $\mathrm{t}$ et $\mathrm{M}_{0}$ correspond à sa masse à l'état initial (échantillon sec).

\subsection{Spectroscopie infrarouge}

\subsubsection{Acquisition des spectres (normal et en mode cinétique)}

Lors d'une analyse par spectroscopie IR à transformée de Fourier en mode réflexion totale atténuée (ATR), l'échantillon est directement mis en contact avec un élément de détection (cristal). Le faisceau infrarouge se réfléchit sur la surface interne du cristal et crée une onde évanescente. Une partie de l'énergie de cette onde est alors absorbée, le rayonnement réfléchi étant renvoyé vers le détecteur (Griffiths et Haseth, 2007). L'un des avantages majeurs de cette technique est la possibilité d'analyser des échantillons sans préparation particulière. En effet, la préparation de pastilles $\mathrm{KBr}$ pour des analyses en transmission est longue et susceptible d'altérer la structure initiale du matériau.

Les analyses ont été menées sur un spectromètre Bruker tensor 27 équipé d'une platine ATR avec un cristal de diamant, d'un détecteur au sulfate de triglycine deutéré RT-DLa TGS et piloté par le logiciel OpusLab v 7.0 122. Les acquisitions ont été réalisées en effectuant 32 balayages entre 4000 et $400 \mathrm{~cm}^{-1}$, avec une résolution de $1 \mathrm{~cm}^{-1}$. Avec ces paramètres, la profondeur de pénétration de l'onde évanescente varie entre $0,4 \mu \mathrm{m}$ et $2,7 \mu \mathrm{m}$ dans le substrat. On a ainsi accès à une information de surface, les faisceaux de fibres ayant quelques dizaines de micromètres d'épaisseur. Les spectres de l'air ambiant utilisés comme référence, ont été obtenus à partir des mêmes réglages. 10 échantillons par fibre et par teneur en eau ont été réalisés dans des conditions de répétabilité. 
Pour la détermination des cinétiques de désorption, des échantillons, conditionnés dans un environnement humide à $95 \%$ d'humidité relative, ont été séchés à l'ambiante sur la platine ATR. Durant le séchage, un spectre IR a été enregistré toutes les 2 minutes avec les mêmes paramètres d'acquisition.

\subsubsection{Traitement des données}

Le spectre de l'air ambiant, ou background, est soustrait à chacun des spectres infrarouges obtenus. Pour le développement des modèles PLS-R (calibration et validation), les spectres bruts ont été utilisés puisque le logiciel optimise les traitements à apporter. Pour le traitement statistique des données, une correction $\mathrm{CO}_{2}, \mathrm{H}_{2} \mathrm{O}$, une correction ATR et un lissage des courbes ont été appliqués. Le calcul de la dérivée seconde de chacun des spectres a également été réalisé. Cette dérivée seconde améliore la résolution des bandes infrarouges ainsi que la discrimination des pics impactés par l'eau. Ces traitements ont été effectués avec le logiciel intégré au spectromètre, à savoir OpusLab v7.0.122.

\subsubsection{Analyses multivariées}

Une régression partielle des moindres carrés (PLS-R) a été réalisée sur les trois types de fibres afin de développer un modèle robuste pour quantifier l'eau présente dans une fibre. L'avantage majeur des modèles multivariés est la prise en compte de l'ensemble des informations du spectre. Le modèle a été réalisé à partir du module QUANT2 (PLS1) du logiciel OPUS ${ }^{\mathrm{TM}}$ v 7.0.122. Pour la calibration, 55 spectres IR contenant les informations spectrales relatives au taux d'humidité de l'échantillon ont été analysés suivant la norme (ASTM Committee E13, 2005). Ces spectres ont été corrélés avec la quantité d'eau réelle contenue dans l'échantillon obtenue par suivi gravimétrique. Les informations statistiques caractérisant cette étape de calibration sont le coefficient de régression linéaire $\mathrm{R}^{2}$ et le RMSECV (erreur quadratique moyenne de la validation croisée). Pour valider le modèle, un jeu de 20 spectres d'échantillons dont la teneur en eau est connue a été testé (validation externe). Les paramètres statistiques ont été ajustés pour obtenir un coefficient de régression linéaire le plus grand possible et le RMSEP (erreur quadratique moyenne de l'erreur de prédiction) le plus faible. Ces paramètres seront commentés dans la partie résultats et discussion.

\section{Résultats et discussion}

\subsection{Analyse qualitative des spectres infrarouges}

La figure 1 représente les spectres ATR-IRTF de fibres de sisal saturées en eau pour différentes conditions d'hygrométrie. Par souci de clarté, seulement 3 humidités relatives $(10,50,85 \%$ pour 3 réplica) ont été représentées. Les valeurs $\mathrm{p}$ (marqueurs ronds sur la figure 1) expriment les nombres d'ondes pour lesquels des changements importants d'absorbance sont observés (obtenus par une analyse de Kruskal Wallis). En d'autres termes, ils permettent d'identifier les régions du spectre impactées par le changement de teneur en eau dans les 
échantillons. Ainsi, même si la totalité du spectre est modifiée par la présence d'eau, l'analyse statistique met en évidence des zones plus affectées que d'autres. Pour une meilleure compréhension du spectre, le tableau 1 regroupe les principales bandes d'absorption impactées par les changements d'humidité.

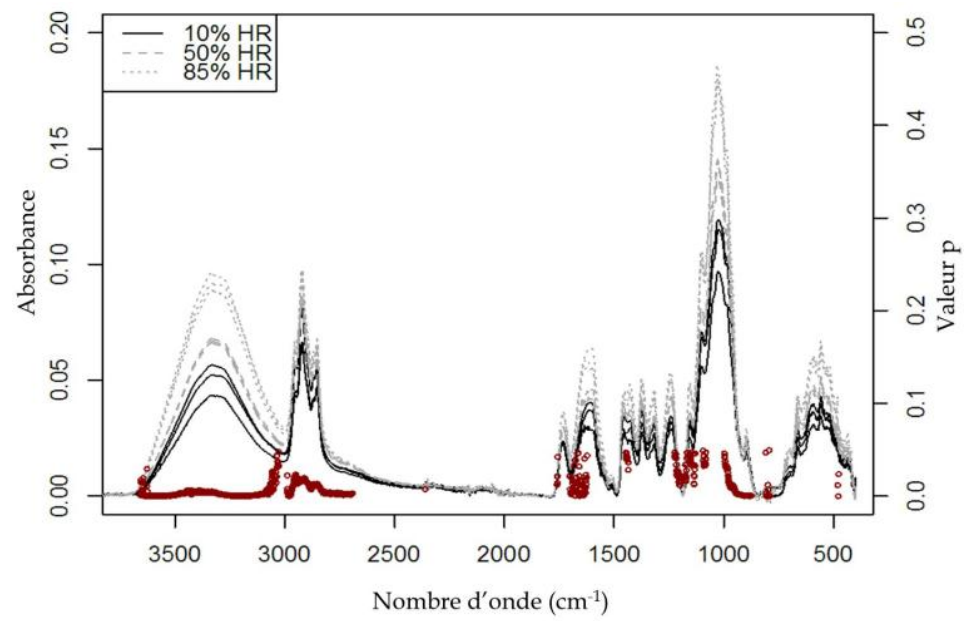

Figure 1. Spectres ATR-IRTF de fibres de sisal saturées en eau à différentes humidités relatives $(-10 \%,-50 \%,--85 \%)$.

Tableau 1. Désignation des principales bandes d'absorption impactées par

l'humidité révélées par l'analyse de variance des spectres infrarouges caractéristiques du sisal (les mêmes pics sont observés pour le lin et le chanvre).

(Fengel, 1992 ; Kondo, 1997 ; Nelsson et O'Connor, 1964)

\begin{tabular}{|c|l|}
\hline $\begin{array}{c}\text { Nombre } \\
\left.\text { d'onde } \mathbf{( c m}^{-1}\right)\end{array}$ & \multicolumn{1}{c|}{ Désignation } \\
\hline $3600-3100$ & $\begin{array}{l}\text { Caractéristique de la vibration d'étirement des liaisons -OH } \\
\text { de la cellulose et de l'hémicellulose }\end{array}$ \\
\hline 2935 & $\begin{array}{l}\text { Étirement des liaisons - } \mathrm{CH} \text { des chaînes de la cellulose } \\
\text { et de l'hémicellulose }\end{array}$ \\
\hline 2862 & Étirement des liaisons - $\mathrm{CH}_{2}$ de la cellulose et de l'hémicellulose \\
\hline 1635 & Vibration en cisaillement de la liaison $-\mathrm{OH}$ de l'eau libre \\
\hline 1425 & Vibration de la liaison carboxyle caractéristique des pectines \\
\hline $1125-895$ & Étirement des liaisons C-O-C de la cellulose \\
\hline 895 & Caractéristique des liaisons $\beta$ de la cellulose \\
\hline $700-650$ & Déformation hors plan de la liaison OH de l'eau \\
\hline
\end{tabular}


La région du spectre la plus impactée par la présence d'eau dans la fibre est la large bande comprise entre 3600 et $3100 \mathrm{~cm}^{-1}$. Pour des matériaux cellulosiques, on a montré par déconvolution, que cette zone représente les liaisons hydrogène du réseau inter et intramoléculaire de la cellulose ainsi que les groupements hydroxyles libres de l'hémicellulose (Fengel, 1992; Hinterstoisser, 1999 ; Nelsson et O'Connor, 1964). Une bande d'absorption dans cette gamme spectrale est également caractéristique de la présence d'eau liquide (Cotugno et al., 2001 ; Murphy et Pinho, 1995). Cette bande représente donc à la fois les interactions macromoléculaires de la cellulose et de l'hémicellulose ainsi que la présence d'eau dans les fibres. Par conséquent, son augmentation significative avec l'absorption d'humidité montre que les molécules d'eau peuvent se fixer sur ces groupements hydroxyles caractéristiques. Une étude plus poussée de ce pic révèle la présence de différents types d'eau plus ou moins liée au réseau macromoléculaire des polymères constitutifs des fibres naturelles.

L'analyse de variance montre aussi un impact significatif de l'eau sur les bandes d'absorption suivantes : 2 944, 2 890,1 635, 1735 et $1425 \mathrm{~cm}^{-1}$ ainsi que la région entre 1100 et $700 \mathrm{~cm}^{-1}$. Les deux premières sont caractéristiques de l'étirement des liaisons - $\mathrm{CH}$. Or, ces groupements étant apolaires, il est peu probable qu'ils constituent des zones préférentielles d'absorption de l'eau. L'hétérogénéité des différents échantillons peut expliquer ce résultat de l'analyse statistique. En effet, chaque spectre étant enregistré pour une fibre différente, il peut exister des écarts induits par l'hétérogénéité de la structure et de la composition des fibres les unes par rapport aux autres. Pour prendre en compte ce facteur dans les modèles, la courbe de calibration sera construite à partir des spectres obtenus dans des conditions de répétabilité, sur 10 échantillons par taux d'humidité relative imposé. Le pic à $1635 \mathrm{~cm}^{-1}$ est assigné à la vibration en flexion de la molécule d'eau. Ce pic témoigne donc directement de la présence d'eau libre dans les échantillons (Kondo, 1997 ; Murphy et Pinho, 1995). Les pics à 1735 et $1425 \mathrm{~cm}^{-1}$, caractéristiques des fonctions carboxyles des pectines constituant la lamelle mitoyenne, sont également impactés par l'absorption d'eau. Ceci met en évidence un deuxième site d'absorption préférentiel des molécules d'eau dans l'échantillon. Enfin, les pics compris entre 1100 à $700 \mathrm{~cm}^{1}$ sont caractéristiques de la nature polysaccharidique de la fibre. Cette signature est également impactée par l'augmentation de l'hygrométrie.

Ces résultats montrent que les spectres contiennent un nombre important d'informations relatives à l'absorption d'humidité. L'utilisation d'un modèle multivarié, prenant en compte l'ensemble du spectre pour quantifier la fraction massique contenue dans ces fibres, semble donc bien adaptée à notre cas d'étude.

\subsection{Analyse quantitative}

\subsubsection{Résultats de l'analyse multivariée}

La fraction massique d'eau contenue dans les trois types de fibres a été évaluée par l'approche de régression partielle des moindres carrés décrite dans la 
section 2.3.3. Un modèle prédictif pour chaque fibre a été développé en utilisant comme valeurs de référence les teneurs en eau expérimentales obtenues par gravimétrie. D'après les méthodes de validation utilisées, ces modèles permettent de prédire des valeurs de teneur en eau contenues dans le domaine de calibration.
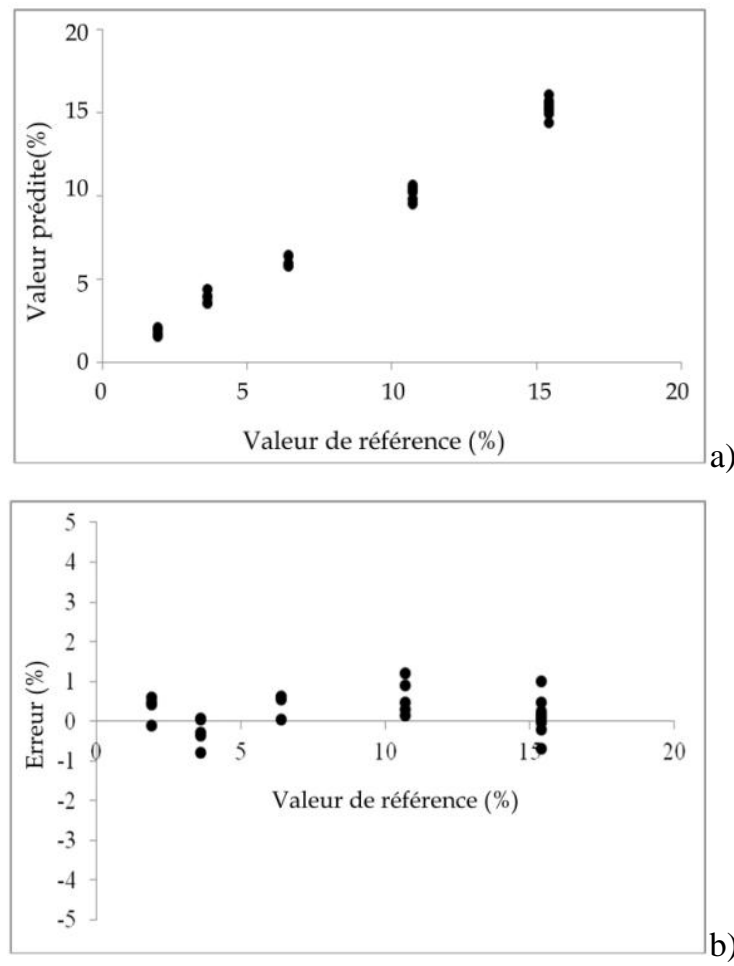

Figure 2. a) fraction massique d'eau prédite par le modèle en fonction de la teneur en eau de référence (obtenue par suivi gravimétrique) pour le sisal (validation externe) b) erreur relative commise sur la valeur de référence

La figure $2 \mathrm{a}$ montre la droite de régression obtenue par validation externe pour la fibre de sisal (tracé de la valeur prédite en fonction de la valeur de référence). La figure $2 \mathrm{~b}$ représente l'erreur commise par rapport à la valeur de référence pour chacune des teneurs en eau prédites. La valeur absolue de cette erreur ne dépasse jamais la valeur limite de $1 \%$. Ces résultats montrent une très bonne corrélation entre valeur prédite et valeur «conventionnellement vraie». Les paramètres statistiques obtenus pour chacune des fibres sont regroupés dans le tableau 2 où on distingue les paramètres obtenus pour la validation croisée et pour la validation externe. Pour chacune des fibres, ces paramètres sont très bons. Les coefficients de régression linéaire élevés associés à des RMSEP et RMSECV faibles témoignent de la robustesse des modèles construits. Toutefois, 
les résultats montrent une meilleure corrélation des modèles pour les échantillons de sisal et de chanvre que pour les échantillons de lin. Ceci, peut s'expliquer par le fait que le matériau lin présente plus de variabilité que les deux autres fibres. Finalement, cette importante corrélation entre valeur prédite et valeur conventionnellement vraie confirme que le spectre IR de fibres humides contient les informations nécessaires pour quantifier la teneur en eau dans les faisceaux de fibres, mettant en évidence la présence de sites hydrophiles mais aussi la présence d'eau libre. L'intérêt de la méthode multivariée est la prise en compte simultanée de l'ensemble de ces sites hydrophiles.

Tableau 2. Paramètres statistiques des modèles PLS-R de chacune des fibres

\begin{tabular}{c|c|c|c|c|}
\cline { 2 - 5 } & \multicolumn{2}{|c|}{ Validation croisée } & \multicolumn{2}{c|}{ Validation externe } \\
\cline { 2 - 5 } & RMSECV & $\mathrm{R}^{\mathbf{2}}$ & RMSEP & $\mathrm{R}^{\mathbf{2}}$ \\
\hline Chanvre & 1,14 & 95,26 & 0,620 & 97,90 \\
\hline Lin & 1,07 & 94,92 & 0,843 & 96,65 \\
\hline Sisal & 0,72 & 98,46 & 0,515 & 99,01 \\
\hline
\end{tabular}

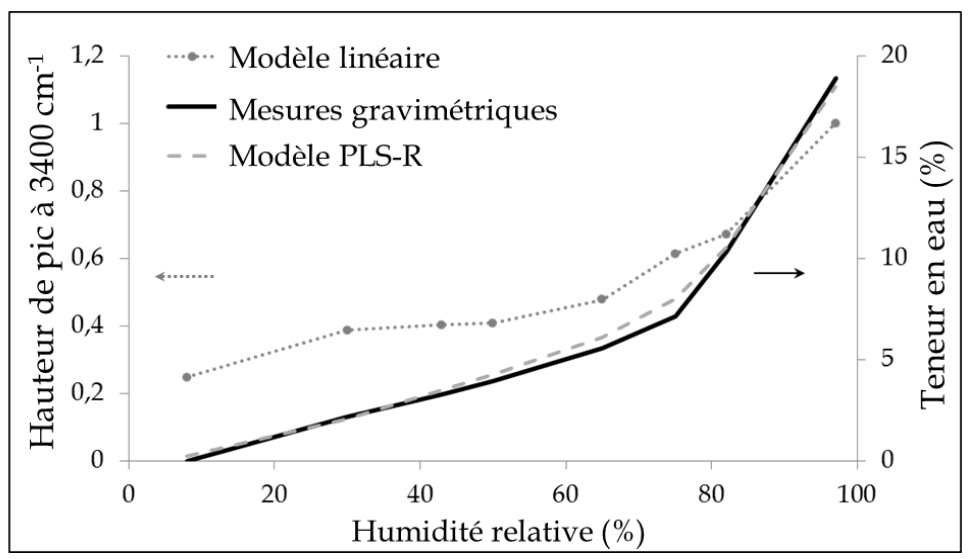

Figure 3. Isothermes de sorption du sisal, obtenues par suivi gravimétrique, par l'utilisation d'un modèle linéaire ou par l'utilisation d'un modèle multivarié

\subsubsection{Validation par les isothermes d'absorption}

Comme indiqué dans la section 2.2., la teneur en eau à saturation des fibres a été déterminée par gravimétrie, pour chaque humidité relative testée. Ceci permet d'accéder aux isothermes d'absorption. Celle du sisal est représentée par la courbe noire sur la figure 3. La quantité d'eau absorbée par la fibre est reliée à l'humidité relative du milieu environnant par une fonction sigmoïdale. Plusieurs 
auteurs rapportent des résultats similaires obtenus sur des polymères poreux (Bessadok et al., 2009; Gouanvé et al., 2007). Cet isotherme s'écarte du modèle de comportement classique des polymères synthétiques (Loos et Springer, 1979). La courbe peut être décomposée en 3 étapes représentées par le modèle de Park. Ce modèle est détaillé dans la référence (Bessadok et al., 2009).

Afin de tester la fiabilité du modèle PLS-R décrit ci-dessus, on a retracé cet isotherme avec les paramètres obtenus. On a également comparé le résultat de ce fitting avec celui d'un modèle linéaire (ou univarié) basé sur la hauteur du pic de vibrations des $-\mathrm{OH}$ à $3400 \mathrm{~cm}^{-1}$. Ces courbes sont représentées respectivement par des traits d'union et des pointillés sur la figure 3. On constate que le modèle linéaire ne donne pas une bonne approximation de l'isotherme d'absorption, en particulier aux faibles humidités relatives. Par l'utilisation d'un tel modèle, Olsson et Salmén (2004) avaient montré une divergence du modèle par rapport aux valeurs de référence à des humidités relatives élevées. Au contraire, le modèle multivarié permet de retracer correctement l'isotherme de sorption du sisal, confirmant sa fiabilité et son utilité vis-à-vis des modèles linéaires.

\subsection{Approche cinétique}

\subsubsection{Comparaison avec les essais gravimétriques}

Une fois ce modèle construit, il doit permettre de déterminer la quantité d'eau d'une fibre dont un modèle de validation externe a été réalisé. Dans cette partie, nous avons ainsi cherché à quantifier la fraction massique d'eau d'une fibre durant son processus de séchage depuis une humidité relative de $95 \%$ jusqu'à l'ambiante $(45 \pm 2 \%)$. Les résultats obtenus par la technique infrarouge ont été comparés à ceux obtenus, dans les mêmes conditions, par suivi gravimétrique en utilisant l'équation (1). La figure 4 présente les résultats obtenus pour le sisal. La cinétique de séchage résultant de la mesure par spectroscopie infrarouge est représentée en noir, alors que le gris correspond au suivi gravimétrique.

Après vieillissement humide à une humidité relative de $95 \%$, les fibres de sisal absorbent $20 \%$ d'eau. Cette teneur en eau concorde avec les résultats obtenus par suivi gravimétrique et avec la littérature (Gouanvé et al., 2007 ; Stamboulis et al., 2001). La courbe de désorption présente une allure sigmoïdale résultant d'un retard de la désorption au départ du processus. Après séchage à l'ambiante, la masse initiale de l'échantillon n'est pas atteinte. On retrouve une perte de masse d'environ $8 \%$ par rapport à la masse initiale. Ceci s'explique par le fait qu'à l'ambiante (HR $=50 \%$ ), la fibre contient une certaine quantité d'eau qui représente une fraction massique de $8 \%$ par rapport à l'échantillon sec.

Les courbes obtenues par la technique de spectroscopie infrarouge et celles obtenues par suivi gravimétrique sont similaires. Une légère différence de pente est observée en régime transitoire. Cet écart peut être attribué au fait que les deux cinétiques n'aient pas été déterminées exactement dans les mêmes conditions. En effet, durant le suivi gravimétrique, l'échantillon est totalement libre, alors que lors de la mesure par spectroscopie infrarouge, les échantillons sont confinés 
sous une pointe permettant d'assurer le contact de l'échantillon avec le substrat. En appuyant sur les fibres, la pointe peut faciliter la désorption et donc induire un séchage légèrement plus rapide que dans l'autre cas.

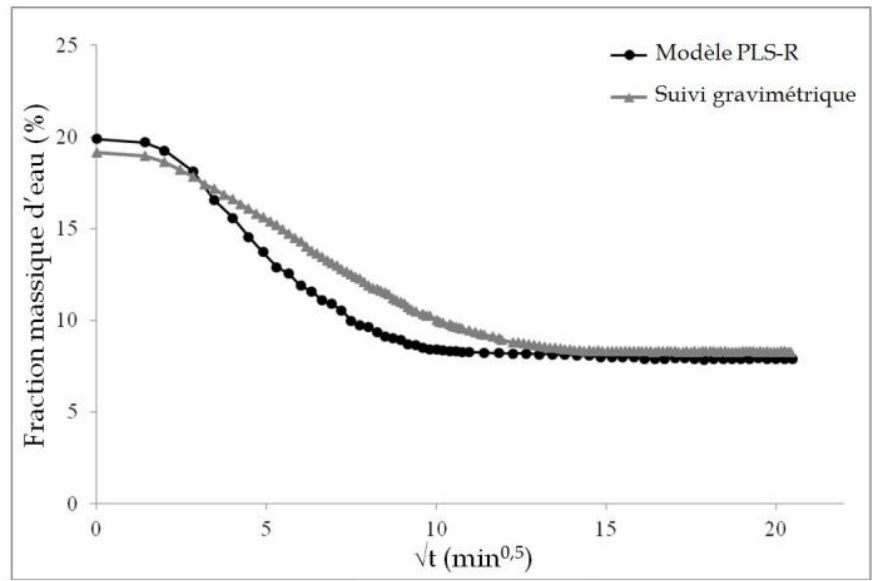

Figure 4. Comparaison des cinétiques de séchage de fibres de sisal obtenues par spectroscopie infrarouge et par gravimétrie

D'après Célino et al. (2013), ce type de courbe peut être décrit par le modèle de Carter et Kibler. Dans ce modèle, on considère que l'eau absorbée dans les fibres se compose de deux phases: une phase mobile et une phase liée. Les molécules de la phase mobile diffusent avec un coefficient de diffusion $\mathrm{D}_{\gamma}$ et se fixent à des sites préférentiels avec une probabilité $\gamma$ par unité de volume. Dans le même temps, les molécules liées peuvent devenir mobiles avec une probabilité $\beta$ par unité de volume. Durant l'absorption ou la désorption d'eau, ces deux phases cohabitent. Comme le souligne la section 3.1., la phase d'eau liée se fixe au réseau macromoléculaire sur des sites spécifiques : les fonctions hydroxyles de la cellulose et de l'hémicellulose ou les fonctions carboxyliques des pectines. L'eau libre s'insère dans la structure poreuse des fibres sous forme de clusters.

Ces résultats montrent que la spectroscopie infrarouge à transformée de Fourier couplée à une analyse multivariée s'avère être un bon outil pour la détermination des cinétiques de diffusion dans les fibres cellulosiques.

\subsubsection{Modifications structurelles}

L'évolution de la structure cristalline des fibres durant le processus de séchage a été étudiée en calculant l'indice total de cristallinité ou TCI décrit par Nelsson et O'Connor dans les années 1960 (Nelsson et O'Connor, 1964). Cette méthode s'appuie sur l'existence, dans le spectre infrarouge de la cellulose, de bandes caractéristiques soit de la cellulose cristalline soit de la phase amorphe. 
On peut estimer la fraction cristalline de la cellulose d'un échantillon en faisant le rapport d'intensité de ces bandes. L'information obtenue est qualitative : elle permet de comparer des échantillons les uns par rapport aux autres.

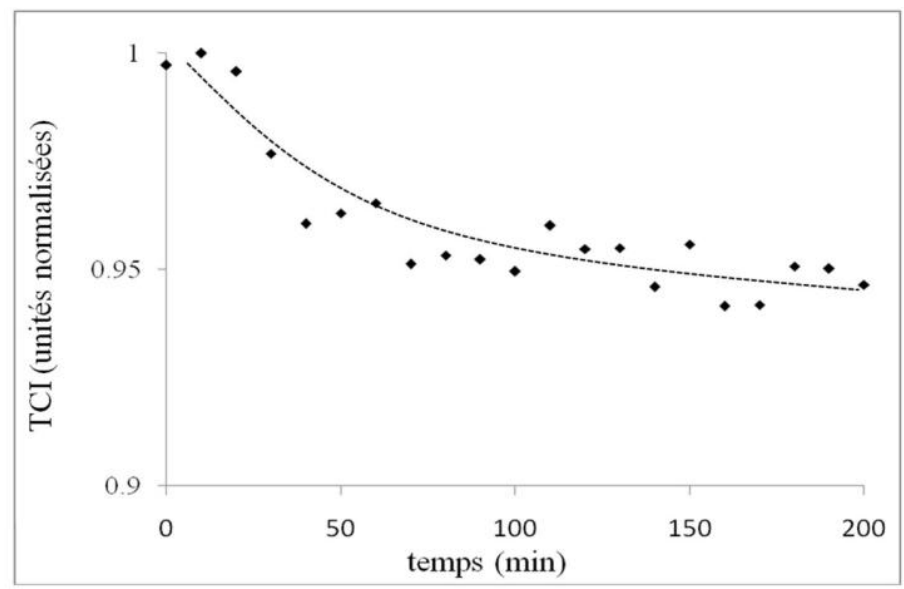

Figure 5. Évolution de la phase cristalline de cellulose contenue dans l'échantillon de sisal durant sa cinétique de séchage

La courbe présentée sur la figure 5 montre l'évolution du TCI au cours de la cinétique de séchage du sisal illustrée sur la figure 4. On observe une diminution de la fraction cristalline de cellulose avec la diminution de la fraction massique d'eau contenue dans l'échantillon. Ceci témoigne d'une action directe de l'eau sur le réseau macromoléculaire de la cellulose. En se retirant de l'échantillon, les liaisons hydrogène créées entre les molécules d'eau et les sites hydrophiles du substrat sont rompues, ce qui a pour conséquence une relaxation du système macromoléculaire et une diminution de la cristallinité de l'échantillon. $\mathrm{Au}$ contraire, lorsque l'eau pénètre dans l'échantillon, la tendance à créer des liaisons hydrogènes avec le substrat entraîne une cristallisation des parties amorphes de la cellulose. Récemment, cette hypothèse a été confirmée par une étude en diffraction des rayons $\mathrm{X}$ de fibres de bois soumises à des cycles de sorption-désorption d'eau (Toba et al., 2013).

\section{Conclusion}

Ce travail souligne plusieurs points particulièrement intéressants concernant l'absorption de l'humidité au sein de fibres cellulosiques à usage de renforts dans les matériaux composites. Ainsi, il a été clairement démontré la présence de sites spécifiques de l'absorption d'eau au sein de ces fibres. Ceci a conduit à l'utilisation d'une approche multivariée pour développer un modèle permettant la quantification de la fraction massique d'eau contenue dans ces fibres. Ce modèle 
a été fiabilisé par l'obtention de paramètres statistiques prouvant une très bonne corrélation entre les valeurs prédites et les valeurs vraies obtenues par gravimétrie. De plus, l'isotherme de sorption caractéristique de ces fibres, qui est bien décrite par le modèle de Park, a été fidèlement retracée par cette méthode. La comparaison avec un modèle linéaire montre l'intérêt d'une approche multivariée exploitant l'ensemble de l'information spectrale. La technique s'est révélée bien adaptée à l'étude des cinétiques de sorption ou désorption de ces fibres. Par rapport aux techniques gravimétriques classiques, elle permet un contrôle in-situ de la diffusion, même aux temps très courts, une automatisation de l'essai et un traitement rapide des résultats. Le calcul de la cristallinité des fibres en fonction de la fraction massique d'eau a mis en évidence un phénomène de cristallisation des échantillons sous chargement humide pouvant apporter une explication quant aux évolutions de propriétés mécaniques observées par certains auteurs (Placet et al., 2012 ; Symington et al., 2009).

\section{Bibliographie}

ASTM Committee E13 on Molecular Spectroscopy and Chromatography. (2005). Standard practices for infrared multivariate quantitative analysis. E 1655-05, p. 1-29.

Bessadok A., Langevin D., Gouanvé F., Chappey C., Roudesli S., Marais S. (2009). Study of water sorption on modified Agave fibres. Carbohydrate Polymers, vol. 76, p. 74$\underline{85}$.

Célino A., Fréour S., Jacquemin F., Casari P. (2013). Characterization and modelling of the moisture diffusion behaviour of natural fibres. Journal of Applied Polymer Science, vol. 130, p. 297-306.

Cotugno S., Larobina D., Mensitieri G., Musto, P. Ragosta, G. (2001). A novel spectroscopic approach to investigate transport processes in polymers: the case of water epoxy system. Polymer, vol. 42, p. 6431-6438.

Dhakal H.N., Zhang Z.Y., Richardson M.O.W. (2007). Effect of water absorption on the mechanical properties of hemp fibre reinforced unsaturated polyester composites. Composites Science and Technology, vol. 67, p. 1674-1683.

Feng J., Berger K.R., Douglas E.P. (2004). Water vapor transport in liquid crystalline and non-liquid crystalline epoxies. Journal of Materials Science, vol. 39, p. 3413-3423.

Fengel D. (1992). Characterization of Cellulose by Deconvoluting the OH Valency Range in FTIR Spectra. Holzforschung, vol. 46, p. 283-288.

Griffiths P.R., Haseth J.A. (2007). Fourier Transformed Infrared Spectrometry, Second edition. John Wiley \& Sons, Inc. p. 321-329.

Gouanvé F., Marais S., Bessadok A., Langevin D., Métayer M. (2007). Kinetics of water sorption in flax and PET fibers. European Polymer Journal, vol. 43, p. 586-598.

Hinterstoisser B., Salmén L. (1999). Two-dimensional step-scan FTIR: a tool to unravel the OH-valency-range of the spectrum of Cellulose I. Cellulose, vol. 6, p. 251-263.

Kondo T. (1997). The assignment of IR absorption bands due to free hydroxyl groups in cellulose. Cellulose, vol. 4, p. 281-292. 
Laity P.R., Hay J.N. (2000). Measurement of water diffusion through cellophane using attenuated total reflectance-Fourier transform infrared spectroscopy. Cellulose, vol. 7 , p. 387-397.

Loos A.C., Springer G.S. (1979). Moisture absorption of graphite/epoxy composites immersed in liquids and in humid air. Journal of Composite Materials, vol. 13, p. 131-146.

Mijovic J., Zhang H. (2003). Local dynamics and molecular origin of polymer networkwater interactions as studied by broadband dielectric relaxation spectroscopy, FTIR and molecular simulations. Macromolecules, vol. 36, p. 1279-1288.

Murphy D., Pinho M.N. (1995). An ATR-FTIR study of water in cellulose acetate membranes prepared by phase inversion. Journal of Membrane Science, vol. 106, p. 245-257.

Musto P., Ragosta G., Mascia L. (2000). Vibrational spectroscopy evidence for the dual nature of water sorbed into epoxy resins. Chemistery of Material, vol. 12, p. 13311341.

Nelsson M.L., O'Connor R.T. (1964). Relation of Certain Infrared Bands to Cellulose Crystallinity and Crystal Lattice Type. Part I. Spectra of Lattice Types I, 11, I11 and of Amorphous Cellulose. Journal of Applied Polymer Science, vol. 8, p. 1325-1341.

Olsson A.M., Salmén L. (2004). The association of water to cellulose and hemicelluloses in paper examined by FTIR spectroscopy. Carbohydrate Research, vol. 339, p. 813818.

Placet V., Cisse O., Boubakar L. (2012). Influence of environmental relative humidity on the tensile and rotational behaviour of hemp fibres. Journal of Materials Science, vol. 47, p. 3435-3446.

Popineau S., Rondeau-Mouro C., Sulpice-Gaillet C., Martin E.R.S. (2005). Free/bound water absorption in an epoxy adhesive. Polymer, vol. 46, p. 10733-10740.

Stamboulis A., Baillie C.A., Peijs T. (2001). Effect of environmental conditions on mechanical and physical properties of flax fibres. Composites Part A: Applied Science and Manufacturing, vol. 32, p. 1105-1115.

Suddell B.C., Evans W.J. (2005). Natural Fiber Composites in Automotive Applications. Natural Fibers in Biopolymers\& Their BioComposites, Editors Mohanty A.K., Misra M. and Drzal L.T., CRC Press, p. 231-259.

Summerscales J., Dissanayake N.P.J., Virk A.S., Hall W. (2010). A review of bast fibres and their composites. Part I-Fibres as reinforcements. Composites Part A: Applied Science and Manufacturing, vol. 41, p. 1329-1335.

Symington M.C., Banks W.M., David W.O, Pethrick R.A. (2009). Tensile Testing of Cellulose Based Natural Fibers for Structural Composite Applications. Journal of Composite Materials, vol. 43, p. 1083-1108.

Toba K., Yamamoto H., Yoshida M. (2013). Crystallization of cellulose microfibrils in wood cell wall by repeated dry and wet treatments, using X-ray diffraction technique. Cellulose, vol. 20, p. 633-643.

Wambua P., Ivens J., Verpoest I. (2003). Natural fibres: can they replace glass in fibre reinforced plastics? Composites Science and Technology, vol. 63, p. 1259-1264. 